Cite this: Phys. Chem. Chem. Phys., 2011, 13, 10491-10502

\title{
Polymer-solid contacts described by soft, coarse-grained models
}

\author{
Marcus Müller, ${ }^{* a}$ Birger Steinmüller, ${ }^{a}$ Kostas Ch. Daoulas, ${ }^{a}$ \\ Abelardo Ramírez-Hernández ${ }^{b}$ and Juan J. de Pablo ${ }^{b}$ \\ Received 13th December 2010, Accepted 8th February 2011 \\ DOI: $10.1039 /$ c0cp02868a
}

The ability of soft, coarse-grained models to describe the narrow interface of a nearly incompressible polymer melt in contact with a solid is explored by numerical self-consistent field calculations and Monte-Carlo simulations. We investigate the effect of the discreteness of the bead-spring architecture by quantitatively comparing the results of a bead-spring model with different number of beads, $N$, but identical end-to-end distance, $R_{\mathrm{e}}$, and a continuous Gaussian-thread model. If the width, $\xi$, of the narrow polymer-solid contact is smaller or comparable to the length of a statistical segment, $b=R_{\mathrm{e}} / \sqrt{N-1}$, strong differences in the interface tension and the density profiles between the two models are observed, and strategies for compensating the discrete nature of the bead-spring model are investigated. Compensating the discretization of the chain contour in the bead-spring model by applying an external segment-solid potential, we simultaneously adjust the interface tension and the density profile to the predictions of the Gaussian-thread model. We suggest that the geometry of the polymer-solid contact and the interface tension are relevant characteristics that a coarse-grained model of polymer-solid contacts must reproduce in order to establish a quantitative relationship to an experimental system.

\section{Introduction}

Polymers in contact with solid boundaries have attracted abiding interest and qualitatively different approaches - ranging from scaling approaches and renormalization group theory to atomistic modeling - have been devised to predict their properties. Using a soft, coarse-grained model, we consider a dense, nearly incompressible polymer melt in contact with a structureless, impenetrable solid substrate and explore the structure and thermodynamics on the length scales ranging from the Edwards screening length, $\xi$, to the size of a polymer coil, $R_{\mathrm{e}}$. Specifically, we explore how a coarse-grained model for a dense polymer melt can cope with the large disparity between the two characteristic length scales, $\xi$ and $R_{\mathrm{e}}$.

The most basic description of the polymer conformations of a melt in contact with a solid boundary-Silberberg's argument ${ }^{1}$ — conceives the effect of the solid to merely consist in mirroring the chain conformations at the polymer-solid contact plane, i.e., the solid imposes a reflecting boundary condition onto the chain conformations.

On the next level of refinement, a compressible melt of very long Gaussian chains in contact with the solid substrate has been considered. ${ }^{2,3}$ The loss of conformational entropy of a

${ }^{a}$ Institut für Theoretische Physik, Georg-August-Universität, 37077 Göttingen, Germany.

E-mail:mmueller@theorie.physik.uni-goettingen.de

${ }^{b}$ Department of Chemical and Biological Engineering, University of

Wisconsin-Madison, Madison, Wisconsin 53706, USA long Gaussian macromolecule in a spatially inhomogeneous environment is described by the Lifshitz entropy ${ }^{4}$ and the finite compressibility of the melt can be modeled by a Helfand compressibility term. ${ }^{5}$ In this case, the free-energy, $F_{\mathrm{GS}}[\phi]$, as a functional of the normalized density, $\phi(\mathbf{r})$, adopts the particularly simple form of the ground-state approximation:

$$
\frac{\mathscr{F}_{\mathrm{GS}}[\phi]}{k_{\mathrm{B}} T \sqrt{\bar{N}}}=\int \frac{\mathrm{d} \mathbf{r}}{R_{\mathrm{e}}^{3}}\left\{\frac{R_{\mathrm{e}}^{2}}{24} \frac{[\nabla \phi(\mathbf{r})]^{2}}{\phi(\mathbf{r})}+\frac{\kappa N}{2}[\phi(\mathbf{r})-1]^{2}\right\}
$$

$\overline{\mathscr{N}} \equiv\left(\rho_{\text {bulk }} R_{\mathrm{e}}^{3} / N\right)^{2}$ denotes the invariant degree of polymerization and $\rho_{\text {bulk }}, N$, and $R_{\mathrm{e}}$ are the segment number density, the number of segments per polymer, and the molecules' end-to-end distance in the bulk, respectively. $\kappa$ is a measure of the inverse compressibility of the dense melt. The equilibrium density profile, which minimizes this free-energy functional, is given by $\mathrm{Wu}$ et $a$. $^{2}$

$$
\phi_{\mathrm{GS}}(x)=\tanh ^{2}\left(\frac{x}{2 \xi}\right)
$$

where the narrow width of the polymer-solid contact is given by the Edwards screening length

$$
\xi=\frac{R_{\mathrm{e}}}{\sqrt{12 \kappa N}}
$$

that describes the range of density fluctuation of the compressible polymer liquid. This length scale is set by a competition between the entropy loss, favoring a broad 
interface, and the non-bonded interactions, minimizing the interface width. These calculations for the Gaussian chain model tacitly assume that the statistics of molecular conformations is Gaussian on the length scale $\xi$, i.e., the statistical segment length, $b_{\mathrm{o}}$, of a polymer molecule is much smaller than $\xi$. Within the ground-state approximation, the interface tension, $\gamma_{G S}$, is given by the analytical expression

$$
\gamma_{\mathrm{GS}}=\frac{2}{3 \sqrt{3}} \frac{k_{\mathrm{B}} T \sqrt{\bar{T}}}{R_{\mathrm{e}}^{2}} \sqrt{\kappa N} .
$$

More accurate theoretical approaches use self-consistent field theory to consider a compressible melt of Gaussian ${ }^{2,3}$ or worm-like chains $^{6-9}$ at a solid substrate. In the self-consistent field theory, ${ }^{5,7,10-16}$ one approximates the interacting multi-chain system by the problem of a single chain in a static, external field, which self-consistently mimics the interaction of a molecule with its surroundings. These mean-field calculations have provided detailed information about the structure and thermodynamics of polymer-solid contacts. According to these calculations, the chain conformations are strongly affected by the inhomogeneous density and the chain ends are enriched in the narrow polymer-solid interface of width $\xi$. Outside of this narrow interface, Silberberg's argument characterizes the leading-order behavior. There are long-range corrections to Silberberg's argument, which have been studied within the mean-field approximation ${ }^{2,3,17}$ and beyond. ${ }^{18-20}$ For instance, there is a depletion of chain ends that extends a distance of order $R_{\mathrm{e}}$ away from the solid. Additionally, the reflection of the molecular conformations at the substrate and the concomitant deepening of the intermolecular correlation hole enhances long-range correlations, ${ }^{19,21,22}$ which cannot be described by self-consistent field theory. These corrections to Silberberg's argument have been studied by Monte-Carlo simulations in thin polymer films. ${ }^{18,20}$

In computer simulations, the structure and thermodynamics of a polymer melt in contact with a solid substrate is often studied by coarse-grained polymer models. The basic idea to derive a coarse-grained model from a chemically realistic description on the atomistic scale consists in lumping $\lambda$ monomeric repeat units of a chemically realistic representation into one coarse-grained segment. ${ }^{23}$ These coarse-grained segments interact via simple and, in our case, soft interactions. The parameters of the soft, coarse-grained model are chosen such that the polymer density, $\rho_{\text {bulk }} / N$, and the spatial extension, $R_{\mathrm{e}}$, of the molecules remain invariant. By virtue of the reduced number of degrees of freedom and the softness of the interactions, coarse-grained models provide a computationally efficient framework for studying structure formation in dense multi-component polymer systems on the length scale from a fraction of $R_{\mathrm{e}}$ to micrometres.

One particular advantage of soft, coarse-grained models ${ }^{24-26}$ is their ability to describe polymer melts with a large invariant degree of polymerization, $\bar{N}$. Let $N_{\mathrm{o}}$ and $N=N_{\mathrm{o}} / \lambda$ denote the number of monomeric repeat units of the chemically realistic model and the coarse-grained representation, respectively. For $R_{\mathrm{e}}$ to remain invariant, the statistical segment length of the coarse-grained model must be $b=R_{\mathrm{e}} / \sqrt{N-1} \approx \sqrt{\lambda} b_{\mathrm{o}}$. Keeping the invariant degree of polymerization fixed, $\overline{\mathcal{N}}=\left(\rho_{\mathrm{o}} b_{\mathrm{o}}^{3}\right)^{2} N_{\mathrm{o}} \approx\left(\sqrt{\lambda} \rho_{\mathrm{o}} b_{\mathrm{o}}^{3}\right)^{2} N$, where $\rho_{\mathrm{o}}$ denotes the number density of monomeric repeat units in the chemically realistic model, one has to increase the number density of coarse-grained segments according to $\rho_{\text {bulk }} b^{3}=\sqrt{\lambda} \rho_{\mathrm{o}} b_{\mathrm{o}}^{3}$. The softness of the potentials allows the coarse-grained segments to overlap, $\rho_{\text {bulk }} b^{3} \gg 1$, which corresponds to a large degree of coarse-graining, $\lambda$, and permits a computationally efficient modeling of large, experimentally relevant values of $\bar{N}$. We note that the expressions for the width of the polymer-solid contact, eqn (2), and the interface tension, eqn (4), do not explicitly depend on the definition of a coarse-grained segment or the detailed form of the interactions between the monomeric repeat units. The variables, $N_{\mathrm{o}}, b_{\mathrm{o}}$ and $\rho_{\mathrm{o}}$, of the chemically realistic model or the quantities, $N, b$ and $\rho_{\text {bulk }}$, of the coarse-grained representation only enter via the coarse-grained parameters, $R_{\mathrm{e}}, \kappa N$, and $\bar{N}$, which will remain invariant if $\lambda$ is altered.

The above arguments suggest that the structure and thermodynamics of the polymer-solid contact is insensitive to the number, $N$, of coarse-grained beads, into which the molecular architecture is discretized and to the detailed form of the interactions between the coarse-grained segments. There are, however, two limitations: (i) The derivation tacitly assumes that the coarse-grained model captures the self-similar, universal Gaussian behavior of the molecular conformations on the scale, $R_{\mathrm{e}}$. This requirement sets a lower limit for the number of coarse-grained segments, $N \gtrsim 16$. If this requirement is not fulfilled, one has to carefully tailor the segmental interactions in order to encode the Gaussian structure of a polymer on large scales. The limiting case, $N=1$ has been carefully studied by Bolhuis and coworkers in the case of polymer solutions. ${ }^{27}$ (ii) The coarse-grained polymer model must capture the molecular architecture on the length scale, $\xi$, of the narrow polymer-solid contact. In the case of dilute or semi-dilute polymer solutions, this is not an additional requirement because the two length scales, $\xi$ and $R_{\mathrm{e}}$, are of the same order of magnitude. In a dense, nearly incompressible melt, however, $\xi / R_{\mathrm{e}} \ll 1$ and the requirement $b \lesssim \xi$ implicates $N \gtrsim 12 \kappa N$, which is very difficult to fulfill by a coarse-grained description.

In this work we explore the consequences of the second limitation on the description of a narrow polymer-solid contact. Specifically, we consider the limit that the statistical segment length, $b_{\mathrm{o}}$, of the chemically realistic description is much smaller than the width, $\xi$, of the polymer-solid contact while $\xi$ is smaller or comparable to the statistical segment length of the coarse-grained representation. In this setting, $b_{\mathrm{o}} \ll \xi \lesssim b$, the coarse-grained model fails to resolve the Gaussian statistics of conformations on the length scale of the polymer-solid contact as illustrated in Fig. 1.

A short overview of this manuscript's structure is the following: In the next section, we briefly summarize the salient features of self-consistent field calculations for arbitrary molecular models and the limiting case of the Gaussian-thread model. By this, we demonstrate that bonded, intramolecular interactions as well as external fields affect the interface tension only via the density profile. In section III, we numerically compute corrections to the ground-state approximation for the interface tension and the density profile of the 


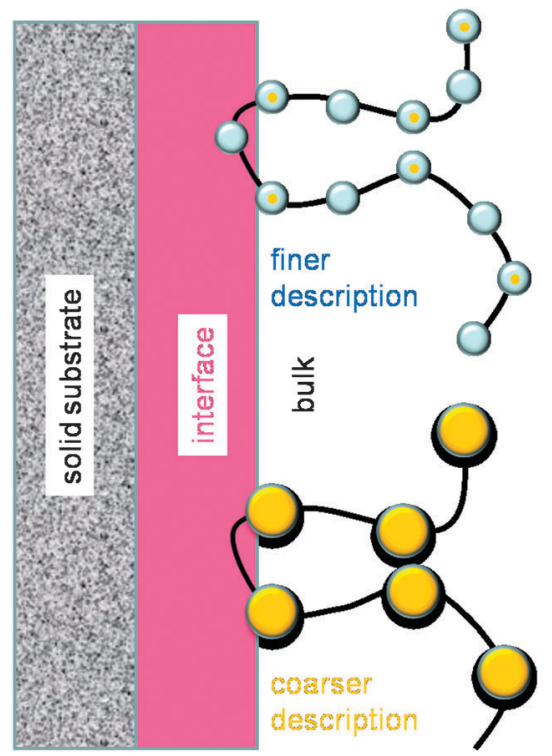

Fig. 1 Sketch of two coarse-grained polymer models with different spatial resolutions in contact with a solid substrate. The chain discretization in the lower representation is coarse, and the size of a coarse-grained segment is comparable to the width of the narrow interface, which is marked in red. In the upper sketch, the molecular contour is discretized into twice as many effective segments, and the structure of the interface is better resolved. While the molecular conformation of the macromolecule on large length scales is identical in the two representations, in the conformation with the lower resolution no effective segment enters the interface while in the finer model one effective segment is located within the interface. In the polymer-solid interface the density decays from its bulk value to zero and the interaction with the neighboring segments, i.e., the nearincompressibility of the melt, gives rise to an effective attraction according to eqn (12). Therefore, the statistical weight of the two conformations will differ.

polymer-solid contact for the Gaussian-thread model and explore the consequences of a large degree of coarse-graining, $\lambda$, on the interface properties. Subsequently, we discuss two methods to compensate for the effects of a coarse molecular representation-(i) altering the pairwise bonded interactions in the vicinity of the solid that has recently been proposed by Ramírez-Hernández et al. $^{28}$ and (ii) adding a segment-solid interaction. The density profile, interface tension and the enrichment of chain ends at the interface are studied. The predictions of the self-consistent field calculations are quantitatively compared to Monte-Carlo simulations of a soft, coarse-grained model. We conclude with a brief summary and comment on the limit where the statistical segment length, $b_{\mathrm{o}}$, of the chemically realistic model is comparable to the interface width, $\xi$.

\section{Model and techniques}

\section{A Self-consistent field theory}

We consider a thin polymer film confined by two impenetrable walls placed at $x=0$ and $x=D$. The interface area of the film is denoted by $A$ and its volume is $V=A D$. The number density of segments, $\hat{\phi}(\mathbf{r})$, at position $\mathbf{r}$ is calculated from the microscopic conformations of the polymer chains according to

$$
\hat{\phi}(\mathbf{r})=\frac{1}{\rho_{\text {bulk }}} \sum_{i=1}^{n} \sum_{t=1}^{N} \delta\left(\mathbf{r}-\mathbf{r}_{i, t}\right)
$$

where the first sum runs over all $n$ polymers in the system and the second sum is taken over all $N$ coarse-grained segments. The position of the $t$ th segment of polymer $i$ is denoted by $\mathbf{r}_{i, t}$. The microscopic density is normalized by the segment number density, $\rho_{\text {bulk }}$. We consider the grandcanonical ensemble, where the thin film is in equilibrium with a reservoir of polymers with chemical potential $\mu$. It is related to the excess activity, $\zeta$, via $\mu=k_{\mathrm{B}} T \ln \zeta+k_{\mathrm{B}} T \ln \left(\rho_{\text {bulk }} /\left[N \mathscr{Q}_{\text {free }}\right)\right.$ where $2_{\text {free }}$ denotes the conformational partition function of a noninteracting, single molecule without geometric constraints. In a thin film, we can distinguish between several different interactions. These are the bonded interactions, $\mathscr{H}_{\mathrm{b}}$, that act along the backbone of a molecule, non-bonded interactions, $\mathscr{F}_{\text {ex }}[\phi]$, that are expressed as a functional of the local density, and an external one-body potential, $V_{\text {wall }} / N$, which represents the interaction of the segments with the confining solid boundaries. With these definitions, the partition function in the grandcanonical ensemble takes the form: ${ }^{29}$

$$
\Xi \propto \sum_{n=0}^{\infty} \frac{e^{\frac{\mu n}{k_{\mathrm{B}} T}}}{n !} \int \prod_{i=1}^{n} \mathscr{P}\left[\{\mathbf{r}\}_{i}\right] e^{-\frac{\sum_{t=1}^{N} V_{\text {wall }}\left(\mathbf{r}_{i, t}\right)}{N k_{\mathrm{B}} T}} e^{-\frac{\mathscr{F}_{\mathrm{ex}}(\hat{\phi})}{k_{\mathrm{B}} T}}
$$

where $\int \mathscr{P}\left[\left\{\mathbf{r}_{i}\right]\right.$ denotes the integral over conformations of molecule, $i$, weighted with the Boltzmann factor of the bonded interactions

$$
\int \mathscr{P}\left[\{\mathbf{r}\}_{i}\right] \cdots=\int \prod_{t=1}^{N} \mathrm{~d} \mathbf{r}_{i, t} e^{-\frac{\mathscr{E} \mathrm{F}_{\mathrm{b}}\left[\mathbf{r} \mathbf{r}_{i}\right]}{k_{\mathrm{B}} T}} \cdots
$$

and $V \mathscr{Q}_{\text {free }}=\int \mathscr{P P}\left[\{\mathbf{r}\}_{i}\right]$.

We rewrite this partition function as a functional integral over the fluctuating density, $\Phi$, and field, $\Omega^{30,31}$

$$
\Xi \propto \int \mathscr{D}[\Phi] \mathscr{D}[\Omega] \exp \left(-\frac{\mathscr{G}[\Phi, \Omega]}{k_{\mathrm{B}} T}\right)
$$

with

$$
\frac{\mathscr{G}[\Phi, \Omega]}{k_{\mathrm{B}} T \sqrt{\bar{N}}}=-\zeta \frac{V}{R_{\mathrm{e}}^{3}} \mathscr{2}[\Omega]+\frac{\mathscr{F} \mathrm{ex}[\Phi]}{k_{\mathrm{B}} T \sqrt{\bar{N}}}-\int \frac{\mathrm{d} \mathbf{r}}{R_{\mathrm{e}}^{3}} \Omega(\mathbf{r}) \Phi(\mathbf{r})
$$

and

$$
V \mathscr{Q}[\Omega]=\frac{1}{\mathscr{Q}_{\text {free }}} \int \mathscr{P}\left[\{\mathbf{r}\}_{i}\right] e^{-\frac{1}{N} \sum_{t=1}^{N} W\left(\mathbf{r}_{i, t}\right)} .
$$

In the last equation, the effective interaction, $W(\mathbf{r})$, that acts on a coarse-grained segment

$$
W(\mathbf{r})=\frac{V_{\text {wall }}\left(\mathbf{r}_{i, t}\right)}{k_{\mathrm{B}} T}+\Omega\left(\mathbf{r}_{i, t}\right)
$$

is comprised of the external segment-solid potential and the fluctuating field, $\Omega$, that represents the non-bonded interactions of a segment.

The mean-field approximation of this partition function is obtained by a saddle-point evaluation of the functional 
integrals over the fields, $\Phi$ and $\Omega$. Their saddle-point values are denoted by $\phi$ and $w$, respectively, with

$$
k_{\mathrm{B}} T w(\mathbf{r})=\frac{N}{\rho_{\text {bulk }}} \frac{\delta \mathscr{F}_{\mathrm{ex}}[\phi]}{\delta \phi(\mathbf{r})}
$$

and

$$
\begin{aligned}
\phi(\mathbf{r}) & =-\zeta V \frac{\delta \mathscr{Q}[w]}{\delta w(\mathbf{r})} \\
& =\frac{\zeta}{\mathscr{Q}_{\text {free }}} \int \mathscr{P}\left[\{\mathbf{r}\}_{i}\right] e^{-\frac{1}{N} \sum_{t=1}^{N} W\left(\mathbf{r}_{i, t}\right)} \sum_{t=1}^{N} \frac{\delta\left(\mathbf{r}-\mathbf{r}_{i, t}\right)}{N} .
\end{aligned}
$$

The last equation identifies $\phi$ as the density that results from an ensemble of mutually non-interacting molecules subjected to the field, $W=V_{\text {wall }} / k_{\mathrm{B}} T+w$. Integrating this last relation over the volume, one obtains

$$
\frac{1}{V} \int \mathrm{d} r \phi(\mathbf{r})=\zeta \mathscr{Q} .
$$

Eqn (12) and (13) constitute a set of self-consistent equations, which are solved via a gradient-free Newton method.

Substituting the saddle-point values, $\phi$ and $w$, back into eqn (9) and expressing the single-chain partition function, $\mathscr{Q}$, in the external field via eqn (14), we can express the mean-field approximation for the grand potential, $G=\mathscr{G}[\phi, w]$, solely as a functional of the density

$$
\begin{aligned}
G= & -k_{\mathrm{B}} T \frac{\rho_{\text {bulk }}}{N} \int \mathrm{d} \mathbf{r} \phi(\mathbf{r}) \\
& +\mathscr{F}_{\mathrm{ex}}[\phi]-\int \mathrm{d} \mathbf{r} \phi(\mathbf{r}) \frac{\delta \mathscr{F}_{\mathrm{ex}}[\phi]}{\delta \phi(\mathbf{r})} .
\end{aligned}
$$

The first term represents the ideal gas contribution, the second term is the excess of the Helmholtz free energy, and the third contribution arises from the Legendre transformation to the grandcanonical ensemble.

From eqn (15) we draw an important conclusion: Since the specific form of the bonded interactions, $\mathscr{H}_{\mathrm{b}}$, or the external one-body potential, $V_{\text {wall }}$, do not enter the grand potential, eqn (15), explicitly but only via the density, $\phi(\mathbf{r})$, we can either modify the bonded interactions, $\mathscr{H}_{\mathrm{b}}$, or the external potential, $V_{\text {wall }}$, or a combination of both in order to compensate for the loss of resolution of the chain contour. If such a correction reproduces the correct density profile it will simultaneously yield the correct interface tension. This is the central result of this section.

In the following, we additionally assume that the range of the non-bonded interactions, which are described by $\mathscr{F}_{\text {ex }}[\phi]$, is short compared to the length scale of the variation of the density profile. This assumption will be appropriate if the range of the non-bonded interactions is comparable to the range of the bonded interactions, $b_{\mathrm{o}}$, in a chemically realistic representation and $b_{\mathrm{o}} \ll \xi$. In this limit, the excess free-energy functional is local

$$
\frac{\mathscr{F}_{\mathrm{ex}}[\phi]}{k_{\mathrm{B}} T}=\frac{\rho_{\text {bulk }}}{N} \int \mathrm{d} \mathbf{r} f(\phi)
$$

and we use Helfand's compressibility term ${ }^{5,54}$

$$
f(\phi)=\frac{\kappa N}{2}(\phi-1)^{2} .
$$

The parameter $\kappa$ is related to the inverse isothermal compressibility via $-\left.V \frac{\partial V}{\partial p}\right|_{n T}=k_{\mathrm{B}} T \rho_{\text {bulk }} \phi(1+\kappa N \phi) / N$. In the following calculations, we set $\zeta=1$ such that the normalized density in the bulk is $\phi_{\text {bulk }}=1$.

The effect of discretization of the molecular contour is investigated using two chain models. First, we use a bead-spring model (BSM), where the bonded interactions are harmonic springs

$$
\frac{\mathscr{H}_{\mathrm{b}, \mathrm{BSM}}\left[\{\mathbf{r}\}_{i}\right]}{k_{\mathrm{B}} T}=\frac{3(N-1)}{2 R_{\mathrm{e}}^{2}} \sum_{t=1}^{N-1}\left[\mathbf{r}_{i, t+1}-\mathbf{r}_{i, t}\right]^{2} .
$$

Second, we take the limit $N \rightarrow \infty$ and define the continuous contour parameter, $0 \leq s=t / N \leq 1$. In this limit, the bead-spring model converges towards the Gaussian-thread model, and the bonded interactions are given by the Edwards Hamiltonian

$$
\frac{\mathscr{H}_{\mathrm{b}, \mathrm{Gauss}}\left[\{\mathbf{r}\}_{i}\right]}{k_{\mathrm{B}} T}=\frac{3}{2 R_{\mathrm{e}}^{2}} \int_{0}^{1} \mathrm{~d} s\left[\frac{\mathrm{d} \mathbf{r}_{i}(s)}{\mathrm{d} s}\right]^{2} .
$$

Technical details of the numerical self-consistent calculations are relegated to the Appendix.

\section{B Monte-Carlo simulations of the bead-spring model}

The self-consistent field calculations invoke a mean-field approximation. In order to assess the accuracy of their predictions we have performed Monte-Carlo simulations of a soft, coarse-grained polymer model, which closely mimics the bead-spring model used in the self-consistent field calculations.

The bonded interactions are identical to eqn (18) used in the self-consistent field calculations. In the Monte-Carlo simulation we express the non-bonded interactions, eqn (16) and (17), through the explicit segment coordinates. Thus, all the interactions depend on the particle coordinates, $\left\{\mathbf{r}_{i, t}\right\}$, and the statistical mechanics of the coarse-grained model can be investigated without any approximation by Monte-Carlo simulation. ${ }^{24}$

To this end, one can either regularize eqn (5) by assigning the particle positions to a collocation lattice ${ }^{25,40,41}$ or calculate the local density in the vicinity of a segment. ${ }^{26,42}$ In the following, we have adopted the latter procedure i.e., instead of eqn (5) we use

$$
\hat{\phi}(\mathbf{r})=\frac{1}{\rho_{\mathrm{bulk}}} \sum_{i=1}^{n} \sum_{t=1}^{N} w\left(\left|\mathbf{r}-\mathbf{r}_{i, t}\right|\right)
$$

where $w(r)=\frac{3}{4 \pi r^{3}}$ for $r \leq r_{\text {int }}=0.0835 R_{\mathrm{e}}$ and $w(r)=0$ otherwise. The range of the weighting function, $r_{\text {int }}$, is dictated by a compromise: (i) $r_{\text {int }}$ is much shorter than the characteristic length scale, $\xi$, of the density profile and therefore it mimics the range of interactions employed in the self-consistent field calculations. (ii) $r_{\text {int }}$ is sufficiently large such that the beads strongly overlap and the soft interactions do not give rise to pronounced liquid-like structuring of the fluid of beads. Under these conditions, the specific form of the weighting function, $w$, is only of minor importance. ${ }^{55}$ 
Using this definition of the local density, we obtain for the non-bonded interactions the following pairwise form:

$$
\mathscr{H}_{\mathrm{nb}}(\{\mathbf{r}\})=\mathscr{F}_{\mathrm{ex}}[\hat{\phi}]=\sum_{(i, t)<(j, s)} V\left(\left|\mathbf{r}_{i, t}-\mathbf{r}_{j, s}\right|\right)
$$

where, in the last step, we have neglected terms that do not depend on the particles' coordinates. The soft pair-potential takes the explicit form:

$$
\begin{aligned}
V\left(\left|\mathbf{r}^{\prime}-\mathbf{r}^{\prime \prime}\right|\right) & =\frac{\kappa k_{\mathrm{B}} T}{\rho_{\text {bulk }}} \int \mathrm{d} \mathbf{r} w\left(\left|\mathbf{r}-\mathbf{r}^{\prime}\right|\right) w\left(\left|\mathbf{r}-\mathbf{r}^{\prime \prime}\right|\right) \\
& =\frac{3 \kappa k_{\mathrm{B}} T}{8 \pi \rho_{\text {bulk }} r_{\text {int }}^{3}}\left(2+\frac{d}{2 r_{\text {int }}}\right)\left(1-\frac{d}{2 r_{\text {int }}}\right)^{2}
\end{aligned}
$$

for $d \leq 2 r_{\text {int }}$ and $V(d)=0$ otherwise, where $d=\mid \mathbf{r}^{\prime}-\mathbf{r}^{\prime \prime}$ denotes the particle distance.

In accord with the previous study ${ }^{28}$ the Monte-Carlo simulations have been performed in the canonical ensemble for a slit with a thickness $D=4 R_{\mathrm{e}}$. We have updated the conformations by local displacements of single segments and slithering-snake movements of complete chains. The parameter combination $N=32, \kappa N=50$, and $\sqrt{\bar{N}}=128$ has been studied.

\section{Results}

\section{A Gaussian-thread model}

In Fig. 2(a) we present the interface tension of a melt in contact with a hard, impenetrable solid as a function of the inverse compressibility of the melt. Even when the chains do not mutually interact, $\kappa N=0$, the presence of the solid boundaries constrains the chain conformations and the interface tension, $\gamma$, is finite. As the inverse compressibility increases, the interface tension increases and for large values of $\kappa N$ we find good agreement with the analytical prediction of the ground-state dominance. ${ }^{2,3}$ In the inset of panel (a), we plot the deviation from the ground-state approximation as a function of $1 / \kappa N$. Such a dependence on chain length has been suggested in previous work. ${ }^{2,3}$ The results of the numerical self-consistent field calculations approach the analytical prediction, $\gamma_{\mathrm{GS}}$, from above according to

$$
\gamma \approx \gamma_{\mathrm{GS}}\left(1+\frac{0.58}{\kappa N}+\cdots\right)
$$

In panel (b) of Fig. 2, we compare the density profiles of Gaussian threads with the prediction of the ground-state approximation. The distance, $x$, from the solid boundary is scaled by the Edwards screening length, $\xi$, in order to compensate for the reduction of the width of the polymersolid interface upon increasing $\kappa N$. For finite values of $\kappa N$, the numerical self-consistent field calculations predict a narrower interface than the ground-state approximation. The main deviation from the density profile, predicted by the groundstate approximation, scales like $1 / \kappa N$ and its range is on the order of $\xi$. This deviation is clearly visible in the central inset, which depicts the scaled relative difference $\kappa N\left(\phi / \phi_{\mathrm{GS}}-1\right)$ versus $x / \xi$. There exists, however, a weak but longer-range
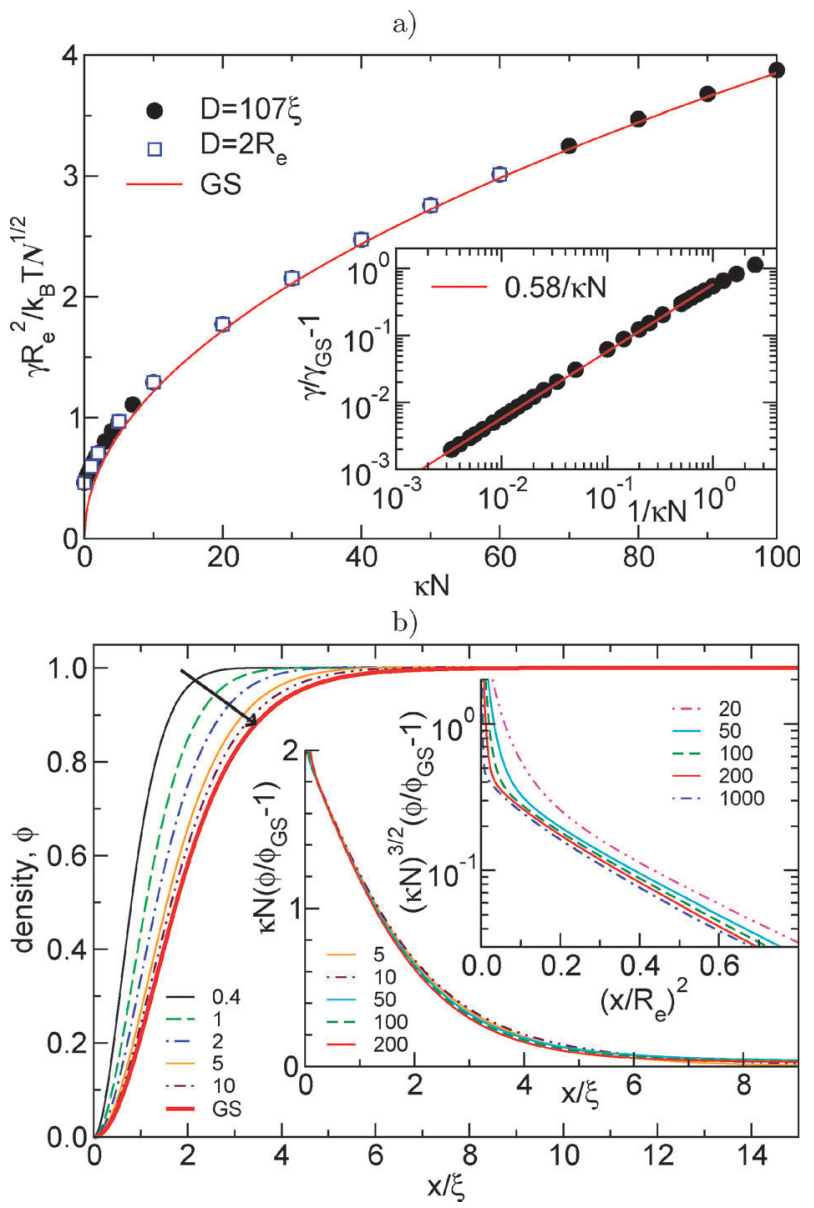

Fig. 2 (a) Scaled interface tension, $\gamma R_{\mathrm{e}}^{2} /\left(k_{\mathrm{B}} T \sqrt{\bar{N}}\right)$, as a function of the inverse compressibility, $\kappa N$, as obtained by self-consistent field calculations of the Gaussian-thread model. The film thickness, $D$, is either kept constant at $D=2 R_{\mathrm{e}}$ or varied according to $D=31 R_{\mathrm{e}} / \sqrt{\kappa N} \approx 107 \xi$. The prediction of the ground-state approximation (GS) is shown for comparison. The inset highlights the correction to the ground-state approximation. (b) Density profiles at a hard, impenetrable solid for various values of the inverse compressibility, $\kappa N$, as indicated in the key. The arrow shows the direction of increasing values of $\kappa N$. The insets highlight the deviation from the ground-state profile.

correction in the tail, which becomes visible when plotting the deviation on a semi-logarithmic scale. A correction of the form $N^{-3 / 2} \tilde{\phi}\left(x / R_{\mathrm{e}}\right)$, with $\tilde{\phi}$ being a scaling function, has been obtained in analytical calculations, ${ }^{2,3,17}$ and our numerical results are compatible with this prediction. Thus the effect of the polymer-solid contact is not completely confined to the narrow interface of width $\xi$, but even within the mean-field approximation, there is a weak correction that extends up to a distance of order $R_{\mathrm{e}}$ away from the solid-the interphase.

\section{B Bead-spring model}

The interface tension, $\gamma$, shown in Fig. 3(a) for various numbers of beads per chain ranging from $N=32$ to $N=16834$, is obtained from the self-consistent field calculations of the bead-spring model. The former value is rather typical for chain discretizations of bead-spring models employed to investigate the structure and thermodynamics of multi-component 
a)

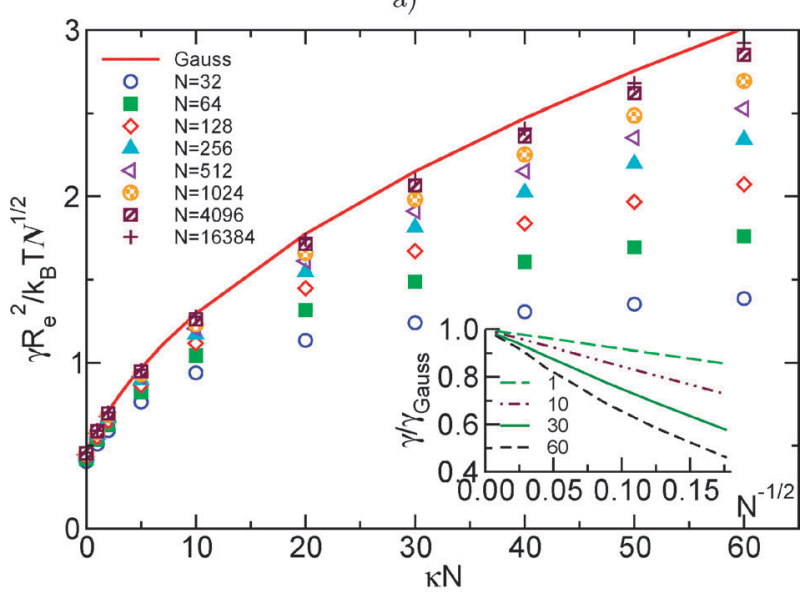

b)

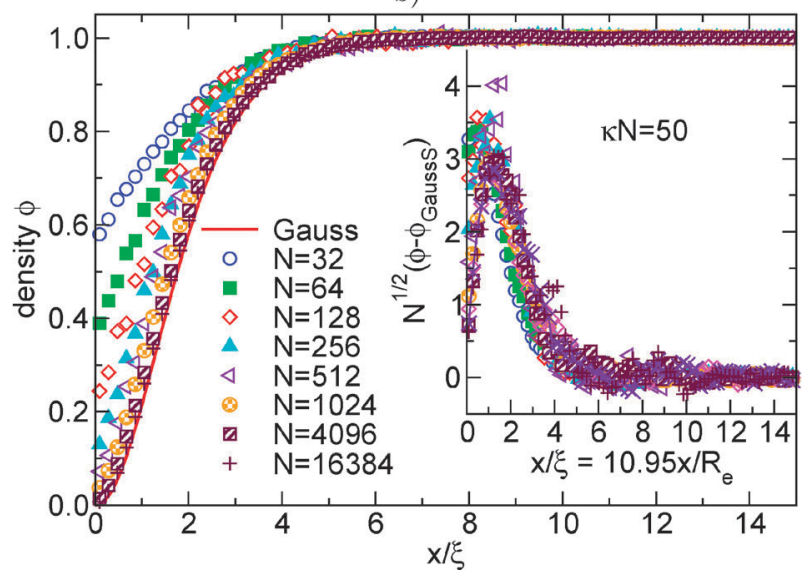

Fig. 3 (a) Scaled interface tension, $\gamma R_{\mathrm{e}}^{2} /\left(k_{\mathrm{B}} T \sqrt{\bar{N}}\right)$, as a function of the inverse compressibility, $\kappa N$, as obtained by self-consistent field calculations of the bead-spring model. The different symbols correspond to a different discretization, $N$, of the chain. The result of Gaussian threads is depicted as a solid line for comparison. The inset demonstrates that the interface tension of the bead-spring models approaches the Gaussian-thread limit for $N \rightarrow \infty$ with a $1 / \sqrt{N}$-correction. The different lines correspond to various values of $\kappa N$ as indicated in the key. (b) Density profiles at a hard, impenetrable solid boundary for various values of $N$, as indicated in the key, and constant $\kappa N=50$. The inset highlights the deviation from the profile of the Gaussian-thread model.

polymer systems on length scale $R_{\mathrm{e}}$. The failure of the coarsegrained model to describe the Gaussian chain statistics on the length scale of the narrow polymer-solid interface results in a spurious dependence of the interface tension on the chain discretization, $N$. The comparison with the result of the Gaussian-thread model ( $c f$. Fig. 2) reveals that results for small $N$ give rise to a significant underestimation of the interface tension. Upon increasing $N$, the results of the bead-spring model gradually approach the data for the Gaussian-thread model representing the limit $N \rightarrow \infty$. The inset demonstrates that the deviation between the bead-spring data for finite $N$ and the results of the Gaussian-thread limit decreases as $1 / \sqrt{N}$.

Fig. 3(b) presents the density profiles, $\phi(x)$, as a function of the distance from the solid boundary. The value of the inverse compressibility is fixed at $\kappa N=50$. In qualitative agreement with previous Monte-Carlo simulations of a soft bead-spring model, ${ }^{28}$ we observe a pronounced $N$-dependence of the density profile in our numerical self-consistent field calculations. For small chain discretization, $N=32$, the density at the solid boundary is overestimated. The inset of panel $b$ shows the scaled deviation of the density profile from the Gaussianthread limit. The amplitude of this deviation decreases like $1 / \sqrt{N}$. Since the graph plots data at fixed $\kappa N$, we cannot distinguish whether the spatial range of the correction is of order $\xi$ or $R_{\mathrm{e}}$. The spatial range appears to be independent of the statistical segment length, $b=R_{\mathrm{e}} / \sqrt{N}=\sqrt{12 \kappa N} \xi / \sqrt{N}$, of the bead-spring model. It approaches its limiting behavior for $N \rightarrow \infty$ from below.

Given this rather pronounced $N$-dependence of the tension and the density profile of the narrow interface, it is tempting to compensate for the effect by either introducing an additional term, $\Delta \mathscr{H}_{\mathrm{b}}$, that modifies the pairwise bonded interactions or by adding an external one-body potential, $V_{\text {wall }}$. Within the mean-field approximation ( $c f$. section II), either strategy can simultaneously adjust the interface tension and the density profile to the known values of the Gaussian-thread model.

\section{Compensation by a modified bonded interaction, $\Delta \mathscr{H}_{\mathbf{b}}$}

In order to derive a modified bonded interaction, $\Delta \mathscr{H}_{\mathrm{b}}$, it is useful to compare the statistical weight, $P_{\mathrm{BSM}}\left(\mathbf{r}_{t+1}, \mathbf{r}_{t}\right)$, of a bond that connects two neighboring beads, $t$ and $t+1$, of a bead-spring model in mean-field approximation

$$
P_{\mathrm{BSM}}=\left(\frac{3(N-1)}{2 \pi R_{\mathrm{e}}^{2}}\right)^{3 / 2} e^{-\frac{3(N-1)}{2 R_{\mathrm{e}}^{2}}\left[\mathbf{r}_{t+1}-\mathbf{r}_{t}\right]^{2}-\frac{w\left(\mathbf{r}_{t+1}\right)+w\left(\mathbf{r}_{t}\right)}{2 N}}
$$

and statistical weight, $P_{\text {Gauss }}$, of a Gaussian thread

$$
\begin{aligned}
P_{\text {Gauss }} & \left.=\int_{r(0)=\mathbf{r}_{t}}^{r\left(\frac{1}{N-1}\right)=\mathbf{r}_{t+1}} \mathscr{D}[r(s)] e^{-\int \mathrm{d} s\left\{\frac{3}{2 R_{e}^{2}}\left[\frac{\mathrm{d} \mathbf{r}}{\mathrm{d} s}\right]^{2}+w(r(s))\right.}\right\} \\
& =q\left(\mathbf{r}_{t+1}, \frac{1}{N-1} \mid \mathbf{r}_{t}\right)
\end{aligned}
$$

that connects the positions, $\mathbf{r}_{t+1}$ and $\mathbf{r}_{t} . q\left(\mathbf{r}_{t+1}, s \mid \mathbf{r}_{t}\right)$ is the partition function of a chain fraction of length $s=\frac{1}{N-1}$ starting at $\mathbf{r}_{t}$ and terminating at $\mathbf{r}=\mathbf{r}_{t+1}$. It also obeys the modified diffusion equation, eqn (37), with the same adsorbing boundary conditions at the film boundaries but with the initial condition

$$
q\left(\mathbf{r}, 0 \mid \mathbf{r}_{t}\right)=\delta\left(\mathbf{r}-\mathbf{r}_{t}\right)
$$

Ramírez-Hernández et al. suggested to $\operatorname{add}^{28}$

$$
\frac{\Delta \mathscr{H}_{\mathrm{b}}(\{\mathbf{r}\})}{k_{\mathrm{B}} T}=-\sum_{t=1}^{N-1} \ln \frac{P_{\mathrm{Gauss}}\left(\mathbf{r}_{t+1}, \mathbf{r}_{t}\right)}{P_{\mathrm{BSM}}\left(\mathbf{r}_{t+1}, \mathbf{r}_{t}\right)}
$$

to the bonded interaction as a way to compensate for the effects of chain discretization. This strategy gives rise to a pairwise bonded interaction that not only depends on the distance between the connected beads but also on their distance from the confining boundaries. In their approach, the field, $w$, which mimics the interaction of a bead with its surrounding neighbors is ignored and the full partition 
function, $q$, of a chain fraction is replaced by the partition function, $q^{(0)}$, of a geometrically confined but otherwise non-interacting chain fraction. This approximation becomes accurate for $\kappa N \rightarrow 0$, i.e., an ideal gas of polymers in contact with a solid. In this limit, the modified diffusion equation can be solved via the method of images. In the case of a semi-infinite system, one obtains ${ }^{28}$

$$
q^{(0)}\left(\mathbf{r}, t \mid \mathbf{r}_{t}\right)=q_{\mathrm{o}}\left(\mathbf{r}, t \mid \mathbf{r}_{t}\right) \frac{e^{-\frac{3\left[x-x_{t}\right]^{2}}{2 R_{\mathrm{e}}^{2} t}}-e^{-\frac{3\left[x+x_{t}\right]^{2}}{2 R_{\mathrm{e}}^{2} t}}}{e^{-\frac{3\left[x-x_{t}\right]^{2}}{2 R_{\mathrm{e}}^{2} t}}}
$$

where

$$
q_{\mathrm{o}}\left(\mathbf{r}, t \mid \mathbf{r}_{t}\right)=\left(\frac{3}{2 \pi R_{\mathrm{e}} t}\right)^{3 / 2} e^{-\frac{3\left[\boldsymbol{r}-\boldsymbol{r}_{t}\right]^{2}}{2 R_{\mathrm{e}}^{2} t}}
$$

is the propagator in free space. Thus the effective interaction energy due to the eliminated degrees of freedom between the coarse-grained segments, $t$ and $t+1$, gives rise to an additional bonding energy of the form ${ }^{28}$

$$
\frac{\Delta \mathscr{H}_{\mathrm{b}}[\{\mathbf{r}\}]}{k_{\mathrm{B}} T}=-\sum_{t=1}^{N-1} \ln \left[1-e^{-\frac{6(N-1) x_{t} x_{t+1}}{R_{\mathrm{e}}^{2}}}\right] .
$$

The spatial range of this correction to the bonded interaction is set by the statistical segment length, $b=R_{\mathrm{e}} / \sqrt{N-1}$, of the coarse-grained bead-spring model. Since it neglects the non-bonded interactions, $\kappa N=0$, it completely ignores the width of the polymer-solid interface, $\xi$.

In Fig. 4 we compare the interface tension of the beadspring model without and with the modified bonded interactions, $\Delta \mathscr{H}_{\mathrm{b}}$, to the results of the Gaussian-thread model. We observe that the interface tension of the augmented beadspring model is much closer to the Gaussian-thread model. For small values of the inverse compressibility, $\kappa N \lesssim 50$, and $N=32$, the difference is less than $5 \%$. For larger values of $\kappa N$ or smaller values of $N$, however, deviations are more pronounced.

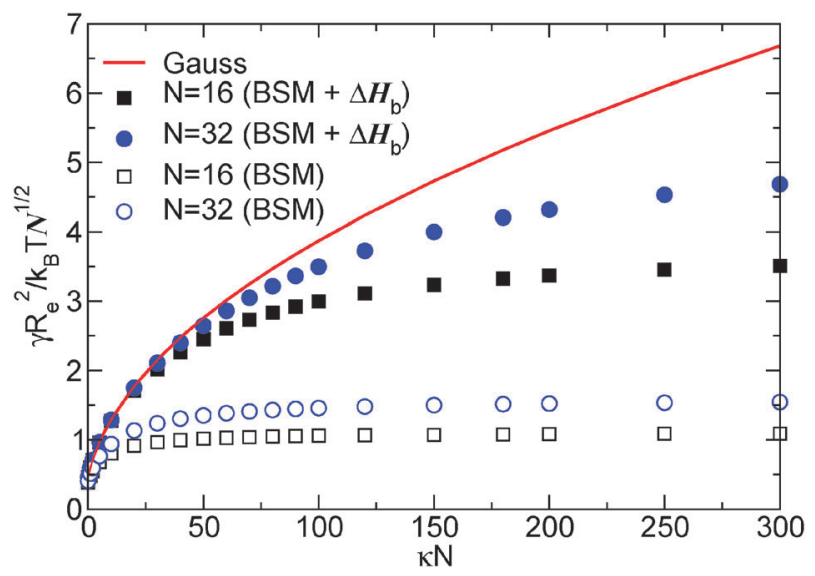

Fig. 4 Scaled interface tension of the Gaussian-thread model, the bead-spring model with $N=16$ and 32, and the bead-spring model with the modified bonded interaction, eqn (31), accounting for geometric confinement.
In order to enhance this method for the problem at hand, one could either treat the field, $w$, as a perturbation ${ }^{2,3}$ and analytically derive an improved estimate for $\Delta \mathscr{H}_{\mathrm{b}}$ or numerically solve the partial differential equation, eqn (37), using the same pseudo-spectral method of the self-consistent field calculations of the Gaussian-thread model. The first strategy is rather cumbersome and remains an approximation. The second avenue is impractical because $\Delta \mathscr{H}_{\mathrm{b}}$ explicitly depends on two variables, $x_{t}$ and $x_{t+1}$, and not only on their difference. If such a numerically determined function were to be used in computer simulations of coarse-grained models, $\Delta \mathscr{H}_{\mathrm{b}}$ would have to be parameterized as a function of two independent variables.

\section{Compensation by an external segment-solid interaction, $V_{\text {wall }}$}

In view of these difficulties it is warranted to explore alternative techniques to compensate for the discretization error. In this section, we will numerically determine an external one-body potential, $V_{\text {wall, }}$ such that the density profile, $\phi(x)$, of the bead-spring model matches the profile, $\phi_{\text {Gauss }}(x)$, of the Gaussian-thread model. Since the density profile is only affected by the combination, $W=w+\frac{V_{\text {wall }}}{k_{\mathrm{B}} T}$, one solves eqn (13) and replaces eqn (12) by the constraint

$$
\phi(x)=\phi_{\text {Gauss }}(x)
$$

for the field, $W(x)$. The external one-body potential that compensates for the discretization effect at the narrow polymer-solid contact is then given by

$$
\frac{V_{\text {wall }}}{k_{\mathrm{B}} T}=W-\frac{N}{k_{\mathrm{B}} T \rho_{\text {bulk }}} \frac{\delta \mathscr{F}_{\mathrm{ex}}[\phi]}{\delta \phi(\mathbf{r})}=W-\kappa N(\phi-1)
$$

Within the mean-field approximation, this external one-body potential, $V_{\text {wall }}$, reproduces the value of the interface tension of the Gaussian-thread model because the grand potential can be expressed as a functional of the density only (cf. eqn (15)). By construction, this strategy does not rely on assuming that the strength of the non-bonded interactions, $\kappa N$, is small.

In Fig. 5 we present the external one-body potential for $\kappa N=50$ and different chain discretizations, $N$. The magnitude of the potential is compatible with a $1 / \sqrt{N}$ behavior but no data collapse occurs when plotting the results versus $x / b$ or $x / \xi$. As expected, both the length scale of the non-bonded interactions, $\xi$, as well as the statistical segment length, $b$, of the coarse-grained bead-spring model are important and no simple scaling of the spatial dependence emerges.

The external segment-solid potential can be obtained with manageable computational effort from one-dimensional self-consistent field calculations, and the numerical results can be parameterized as a function of the distance, $x$, from the polymer-solid contact. Alternatively, the mean-field approximation can be avoided by using computer simulations, and $V_{\text {wall }}$ can be numerically obtained by iterative Boltzmann inversion $^{38}$ of eqn (32). Calculating the density profile and the pair correlation function of density fluctuations at the solid in the simulations, one can predict the change 


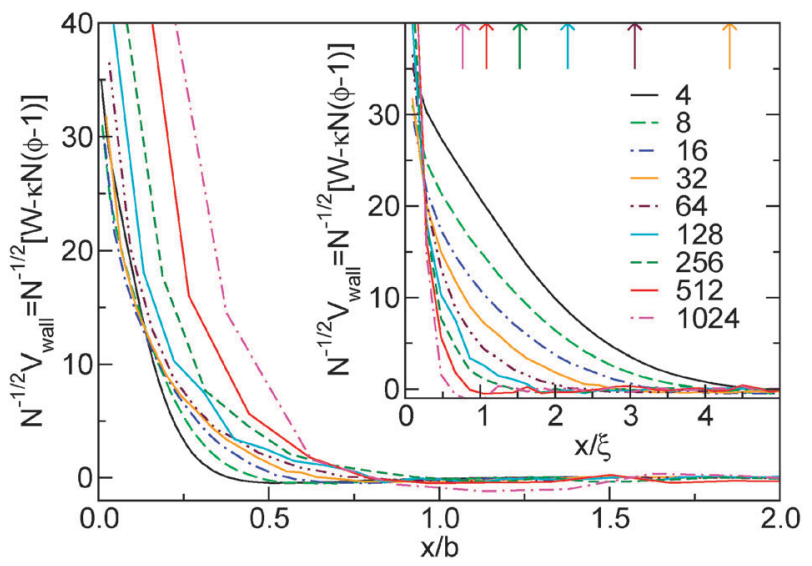

Fig. $5 V_{\text {wall }} / k_{\mathrm{B}} T$ as a function of the distance, $x$, from the wall. The inverse compressibility is $\kappa N=50$ and the different values of the chain discretization, $N$, are indicated in the key. The amplitude of the compensation potential that acts on a segment is compatible with a $1 / \sqrt{N}$-behavior. The main panel plots the scaled potential as a function of $x / b=x \sqrt{N} / R_{\mathrm{e}}$ while the inset presents the scaled potential versus $x / \xi$. The arrows on the top mark $b / \xi \approx \sqrt{\frac{12 \kappa N}{N}}$.

of the interface tension and the density profile in response to changing $V_{\text {wall: }}{ }^{39}$

$$
\begin{aligned}
\frac{\delta G}{\delta V_{\text {wall }}(\mathbf{r})} & =\frac{\rho_{\text {bulk }}}{N}\langle\hat{\phi}(\mathbf{r})\rangle \\
\frac{\delta \phi(\mathbf{r})}{\delta V_{\text {wall }}\left(\mathbf{r}^{\prime}\right)} & =-\frac{\rho_{\text {bulk }}}{N k_{\mathrm{B}} T}\left(\left\langle\hat{\phi}(\mathbf{r}) \hat{\phi}\left(\mathbf{r}^{\prime}\right)\right\rangle-\langle\hat{\phi}(\mathbf{r})\rangle\left\langle\hat{\phi}\left(\mathbf{r}^{\prime}\right)\right\rangle\right)
\end{aligned}
$$

\section{E End segment distribution}

Adding an appropriate $V_{\text {wall }}$ we exactly reproduce the density profile and the interface free energy. This encourages us to study the behavior of other characteristics of the polymer melt in contact with the solid. In Fig. 6(a) and (b) we illustrate the influence of $\Delta \mathscr{H}_{\mathrm{b}}$ and $V_{\text {wall }}$ on the spatial distribution of chain ends for $\kappa N=50$ and 100 , respectively. The main panels depict the relative excess of chain ends, $\Delta \phi_{\text {end }}(x)=N \phi_{\text {end }}(x) / 2-$ $\phi(x){ }^{2}$ In the Gaussian-thread model, chain ends are enriched at the interface and there is a concomitant depletion zone that extends a distance $R_{\mathrm{e}}$ away from the solid., ${ }^{2,3} \mathrm{We}$ note that the bead-spring model with a contour discretization of $N=32$ overestimates the density of chain ends at the polymer-solid interface in comparison with the predictions of the Gaussian-thread model. ${ }^{56}$ In fact, the absolute value of $\phi_{\text {end }}(x)$ in the bead-spring model remains almost constant for $\kappa N=50$ or even increases towards the surface for $\kappa N=100$. For the Gaussian-thread model, however, both the segment density as well as chain end density vanish at the polymersolid contact, $x=0$.

A modification of the bonded interactions in the vicinity of the surface qualitatively reproduces the behavior of the Gaussian-thread model, i.e., $\Delta \phi_{\text {end }} \rightarrow 0$ for $x \rightarrow 0$, a positive excess of chain ends in the narrow interface, and a concomitant depletion in the broader interphase. However, the magnitude of the enrichment in the interfaces and the depletion in the range $\xi \lesssim x \lesssim R_{\mathrm{e}}$ are overestimated, and the deviation from the prediction of the Gaussian-thread model increases as the a)

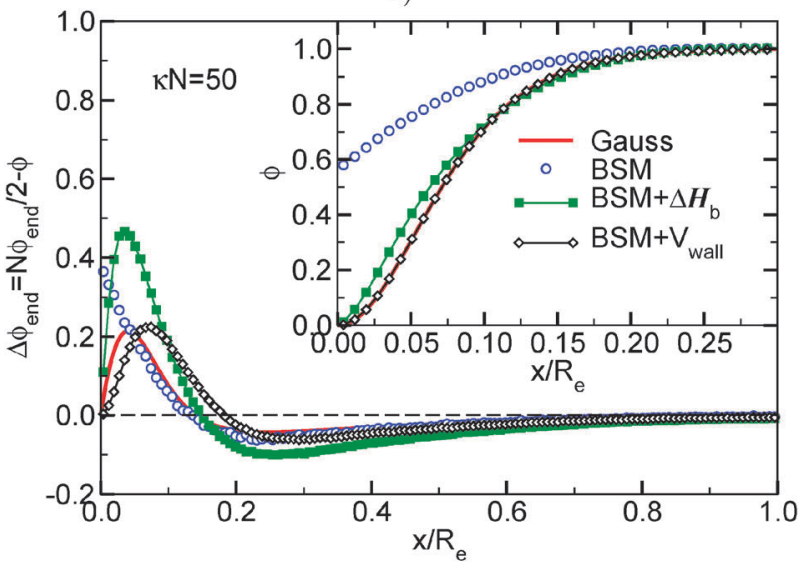

b)

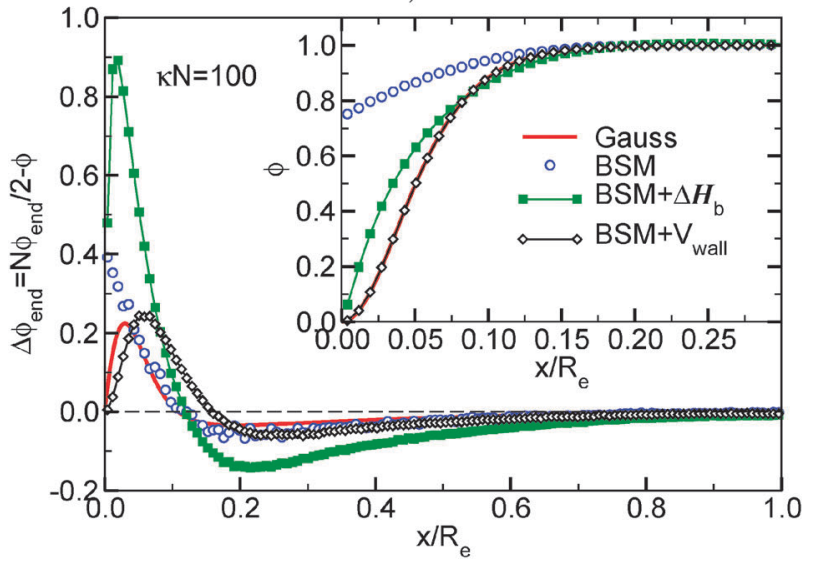

Fig. 6 Distribution of chain ends at a polymer-solid contact. The main panels present the relative excess of chain ends, $\Delta \phi_{\text {end }}(x)$, for (a) $\kappa N=50$ and (b) $\kappa N=100$, respectively. The predictions for the Gaussian-thread and bead-spring models without compensation, with modified bonded interaction, $\Delta \mathscr{H}_{\mathrm{b}}$, and with an external segmentsolid interaction, $V_{\text {wall }}$, are shown. $N=32$ beads are used to represent the molecular contour in the bead-spring models. The insets depict the density profiles for comparison.

melt becomes less compressible. Using $V_{\text {wall, }}$ we do not observe perfect agreement with the prediction of the Gaussianthread model either but the results are significantly closer to the model with infinitely fine discretization than the bare bead-spring model or the model with modified bonded interactions. The advantage of compensating the chain discretization by an external segment-solid interaction, $V_{\text {wall }}$, becomes more pronounced for the larger value of $\kappa N$, where already the density profile with $\Delta \mathscr{H}_{\mathrm{b}}$ clearly deviates from the Gaussianthread model.

\section{Comparison to Monte-Carlo simulations of a soft, coarse-grained model}

To take into account the influence of the solid, we have applied both approaches: (i) we included a modified bonded interaction between beads ( $c f$. eqn (31)) and (ii) we included an external segment-solid interaction obtained by using selfconsistent field theory. 
The external segment-solid interaction is presented in Fig. 7a. The different results were obtained by self-consistent field calculations in the grandcanonical ensemble ( $c f$. Fig. 5) for $D=2 R_{\mathrm{e}}$ and canonical self-consistent field calculations for $D=4 R_{\mathrm{e}}{ }^{57}$ In order to obtain the latter result, we have first calculated the density profile for the Gaussian-thread model in a film of thickness $D=4 R_{\mathrm{e}}$ and used this density profile $\phi_{\text {Gauss,can }}$ in the canonical ensemble to calculate $w$ for the bead-spring model. The external one-body potential is obtained by $V_{\text {wall }} / k_{\mathrm{B}} T=W-\kappa N\left(\phi_{\text {Gauss,can }}-1\right)+c$, where the constant, $c=2.09101$, ensures that $V_{\text {wall }} \rightarrow 0$ for $x \rightarrow \infty$. Fig. 7(a) shows that the external potentials, $V_{\text {wall }}$, obtained in the grandcanonical and the canonical ensemble are very similar. In the Monte-Carlo simulations we have used the potential $V_{\text {wall }} / k_{\mathrm{B}} T=W-\kappa N\left(\phi_{\mathrm{GS}}-1\right)$ where $W$ has been calculated in the grandcanonical ensemble. At short distances, $x<0.1 R_{\mathrm{e}}$, the potential is strongly repulsive and it includes a small attractive component at intermediate distances.

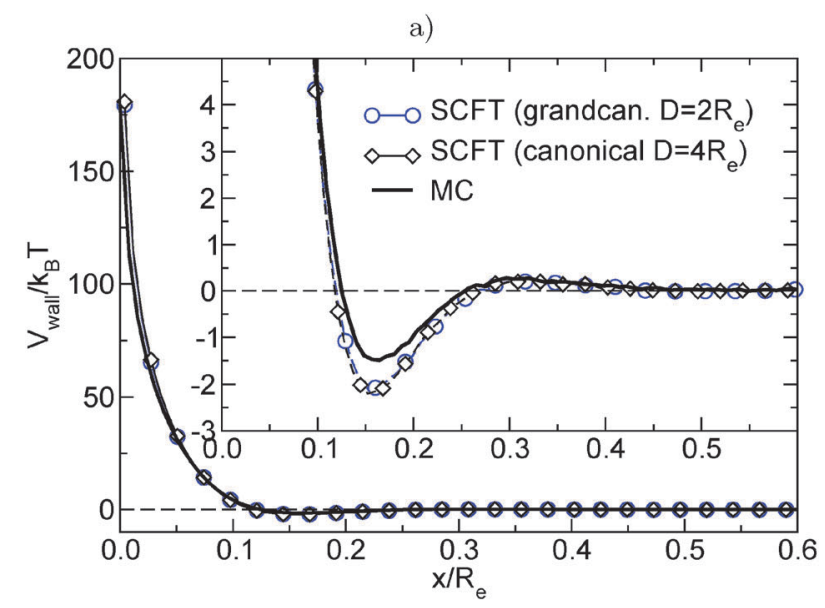

b)

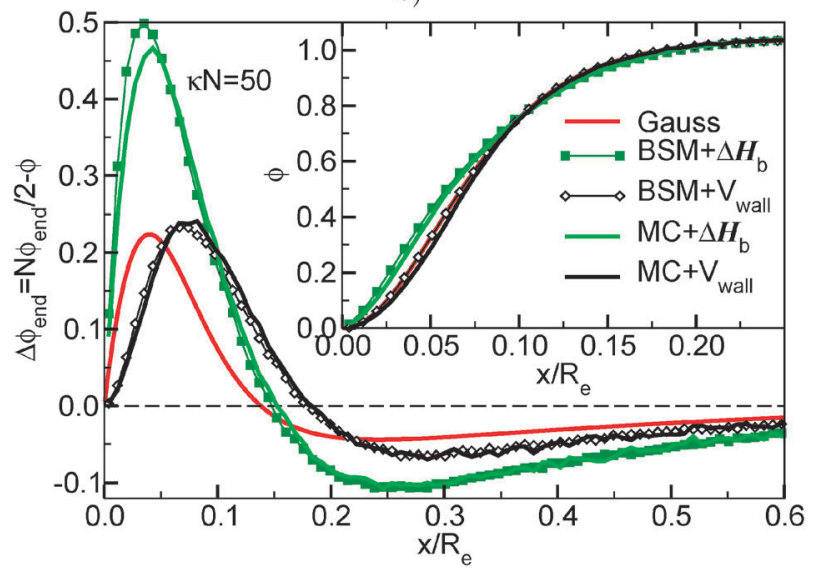

Fig. 7 (a) $V_{\text {wall }} / k_{\mathrm{B}} T$ as a function of the distance, $x$, from the wall for $\kappa N=50, \sqrt{\bar{N}}=128$, and chain discretization $N=32$. The panel presents the predictions of the self-consistent field theory in the grandcanonical and canonical ensemble and the potential, which has been utilized in the Monte-Carlo simulation. (b) Excess of chain ends and density profile (inset) at the polymer-solid contact as obtained by self-consistent field calculations in the canonical ensemble $D=4 R_{\mathrm{e}}$ and Monte-Carlo simulations. Results using the modified bonded interaction and the external segment-solid interaction are shown.
The quantitative comparison between the results of the Monte-Carlo simulations and the self-consistent field theory is presented in Fig. 7. The main panel presents the excess of chain ends at the polymer-solid contact and the inset depicts the density profiles. First we note that the predictions of the self-consistent field theory and the results of the Monte-Carlo simulations are in very good, quantitative agreement without any adjustable parameter. This illustrates (i) the ability of soft, coarse-grained models to establish a quantitative relation between particle-based models and field-theoretic models and (ii) the accuracy of the mean-field approximation for polymer systems with a large invariant degree of polymerization, $\bar{N}$. Second, in agreement with the predictions of the previous section, both approaches improve the behavior of the monomer density near walls. Using the external potential, we obtain a better quantitative agreement with the theoretical results of Gaussian-thread model for the density of chain ends.

\section{Summary and conclusions}

Using self-consistent field calculations of a Gaussian-thread and a bead-spring model, we have studied the properties of a polymer-solid contact in the regime where the chemically realistic conformations of a macromolecule obey Gaussian statistics on the length scale of the narrow polymer-solid contact, $\xi$. Within the mean-field approximation, the interface tension can be expressed solely as a functional of the density profile, $\phi(x)$; it depends on the bonded interactions or interaction with the wall (or an external field) only via the density.

For the Gaussian-thread model, we have numerically derived a first-order correction to the ground-state approximation for the interface tension, eqn (24), and illustrated that the density profile exhibits a small correction that persists up to distances of the order $R_{\mathrm{e}}$ away from the solid. This finding is in agreement with previous analytical considerations. ${ }^{2,3}$

We have shown that chain discretization effects will have a rather pronounced impact on the density profile and the interface tension if the statistical segment length, $b=R_{\mathrm{e}} / \sqrt{N-1}$, of the bead-spring model becomes comparable to or larger than the width of the narrow polymer-solid contact, $\xi$. For a small discretization, $N$, of the molecular contour, the density at the polymer solid contact is overestimated and the interface tension is underestimated. Two strategies for compensating these discretization artifacts have been explored-modifying the pairwise bonded interactions, $\Delta \mathscr{H}_{\mathrm{b}}$, in the vicinity of the solid and augmenting the model with an external one-body potential, $V_{\text {wall }}$. In the former case, a simple analytical expression has previously been derived for $\Delta \mathscr{H}_{\mathrm{b}}$ ignoring the non-bonded interactions, i.e., $\kappa N=0 .{ }^{28} \mathrm{We}$ show that this approximation yields accurate estimates for the surface tension up to $\kappa N \lesssim 50$ for $N=32$. For larger values of the inverse compressibility or smaller chain discretizations, however, the deviations from the predictions of the Gaussianthread model grow substantially. In the latter case, the external one-body potential, $V_{\text {wall }}$, can be obtained using numerical self-consistent field theory at low computational expense. In contrast to $\Delta \mathscr{H}_{\mathrm{b}}, V_{\text {wall }}$ only depends on the distance of the coarse-grained segment from the solid boundary and can be easily parameterized for its use in numerical 
simulations. Naturally, the same form of an external one-body potential, $V_{\text {wall }}$, can also account for explicit interactions between the polymer and the solid, for example a long-range van-der-Waals interaction, which have not been considered in the present study. Moreover, in situations where the density profile at the polymer-solid contact or the interface tension are unknown, such an external one-body potential also provides a convenient means to adjust the interface tension in a coarsegrained representation to experimentally measured values.

Lastly, a comment on the assumed validity of the Gaussianthread model is in order. Throughout the manuscript we have assumed that the statistical segment length, $b_{\mathrm{o}}$, of a chemically realistic chain is smaller than the Edwards screening length, $\xi$, such that the "true" molecular statistics on the length scale $\xi$ is Gaussian. In semi-dilute polymer solutions this assumption may be questionable because the Edwards screening length, $\xi$, also sets the length scale of the cross-over between selfavoiding chain statistics on length scales smaller than $\xi$ and Gaussian chain behavior for larger length scales. The width of the polymer-solid contact is comparable to the cross-over length scale and corrections to the Gaussian chain statistics have to be anticipated. In dense polymer melts, in turn, the screening length may become comparable to $b_{\mathrm{o}}$ itself, and the discrete molecular structure of the chemically realistic macromolecule on the length scale, $\xi$, becomes important. In both cases, the physical properties of the interface are not completely described by the coarse-grained parameters, $R_{\mathrm{e}}, \kappa N$, and $\overline{\mathcal{N}}$, even for very fine chain discretizations. Local details of the molecular architecture (e.g., molecular stiffness or local swelling of the chains due to excluded volume) and the fluidlike packing of the monomeric repeat units may exert a pronounced influence on the interface. The discretization effects discussed in the present manuscript may qualitatively indicate some aspects of the physical consequences of the discrete molecular structure on the properties of the narrow polymer-solid contact. Additional effects-e.g., stiffness along the molecular backbone, shape of the monomeric repeat units and their liquid-like packing in a dense melt, and a more realistic equation of state - have to be considered to complete the picture of polymer-solid contacts. A more quantitative description can be achieved by polymer density-functional theory. ${ }^{43-51}$

Even if a highly coarse-grained model cannot describe the detailed properties of the polymer-solid contact, one may still contemplate the issue, which the relevant properties of a polymer-solid interface are that a coarse-grained model has to capture in order to describe the properties on the larger length scale $R_{\mathrm{e}}$. Certainly, the geometry of the solid (area and shape) as well as the tension constitute a minimal set of relevant characteristics of polymer-solid contacts because they dictate fundamental thermodynamic properties (e.g., the stability of a polymer film in contact with the solid against de-wetting). For instance, coarse-grained simulations of blends of diblock copolymers and nano-particles ${ }^{52}$ illustrated that the shape and size of the nano-particle boundary and its interface tension are sufficient to predict the rather subtle effects that dictate the distribution of the nano-particle in the spatially modulated polymer system. The geometry of the solid and its interface tension are also experimentally accessible (e.g., by contact angle measurements). These coarse-grained interface parameters play a similar role as the end-to-end distance, $R_{\mathrm{e}}$, in the bulk in establishing a quantitative relation between a coarse-grained model and an experimental system. They cannot be predicted by a coarsegrained representation but once the coarse-grained model has been adjusted to reproduce the experimental value, we expect it to be useful for predicting properties on larger length scales.

Additional refinements may aim for adjusting the length scale of the segment-solid interaction (i.e., short-range, exponential decay versus a power-law decay due to van-der-Waals interactions) because it may qualitatively affect the wetting behavior and stability of thin films. ${ }^{53}$ The range and strength of the interaction may also have a pronounced effect on the single-chain dynamics, since a strongly attractive, shortranged potential will tend to strongly adsorb the molecules onto the solid and lead to protracted exchange kinetics between the interface and the bulk. These coarse-grained properties of the polymer-solid contact can be conveniently introduced in a computational model via a segment-solid interaction, $V_{\text {wall }}$.

\section{Appendix A: details of the numerical self-consistent field calculations}

\section{Partial enumeration technique for the bead-spring model}

In order to calculate the partition function, $\mathbb{Q}$, of a single chain in the field $w$ and its functional derivative, we resort to a partial enumeration scheme $\mathrm{e}^{32-36}$ where one approximates the integral over all chain conformations by the sum over a large number, $n_{c}$, of conformations, $\boldsymbol{r}_{c, t}$. These single-chain conformations are generated according to the Boltzmann factor, $\exp \left(-\frac{\mathscr{H}_{\mathrm{b}, \mathrm{BSM}}\left[\{\mathbf{r}\}_{c}\right]}{k_{\mathrm{B}} T}\right)$, i.e., they are representative of non-interacting, single molecules in the bulk. In this framework, one obtains

$$
\mathrm{Q}=\frac{1}{n_{c}} \sum_{c=1}^{n_{c}} e^{-\frac{\Delta \mathscr{H}_{\mathrm{b}}}{k_{\mathrm{B}} T}-\sum_{t=1}^{N} \frac{W\left(\mathbf{r}_{c, t}\right)}{N}}
$$

where $\Delta \mathscr{H}_{\mathrm{b}}$ represents additional bonded interactions, which may be introduced to compensate discretization effects ( $c f$. section IIIC). We calculate the density according to eqn (13) using

$$
-V \frac{\delta \mathscr{Q}[w]}{\delta w(\mathbf{r})}=\frac{1}{n_{c}} \sum_{c=1}^{n_{c}} e^{-\frac{\Delta \mathscr{H}_{\mathrm{b}}}{k_{\mathrm{B}} T}-\sum_{t=1}^{N} \frac{W\left(\mathbf{r}_{c, t}\right)}{N}} \sum_{t=1}^{N} \frac{V \delta\left(\mathbf{r}-\mathbf{r}_{c, t}\right)}{N}
$$

Typically, we use $n_{c}=10^{7}$. The evaluation of the single-chain properties is performed by dividing the set of single-chain conformations equally among different processors independently of their position in space. Cores compute the contribution of "their" conformations to $\mathscr{Q}$ and $\phi$ in parallel and the results are summed across the different processors. ${ }^{34-36}$ This computational scheme can be generalized to arbitrary single-chain conformations and allows us to incorporate details of the molecular architecture on small scales (e.g., stiffness along the molecular backbone or non-linear chain topologies). 


\section{Gaussian-thread model}

It is useful to define a partition function, $q(\mathbf{r}, s)$, of a chain fraction of length $s$ starting anywhere in the volume, $V$, and terminating at position $\mathbf{r}$. It obeys the modified diffusion equation $^{5}$

$$
\frac{\partial q}{\partial s}=\frac{R_{\mathrm{e}}^{2}}{6} \Delta q-w q
$$

with the starting condition

$$
q(\mathbf{r}, s=0)=1
$$

and the boundary conditions ${ }^{58}$

$$
q(x=0, y, z, s)=0 \text { and } q(x=D, y, z, s)=0 .
$$

We solve this partial differential equation using a pseudospectral method. ${ }^{37}$ The integration along the chain contour is very finely discretized with $\Delta s=1 / 16324$ in order to accurately represent the limit of continuous Gaussian threads. Once $q(\mathbf{r}, s)$ is obtained, one can calculate the single-chain partition function

$$
\mathscr{Z}=\frac{1}{V} \int \mathrm{d} \mathbf{r} q(\mathbf{r}, s) q(\mathbf{r}, 1-s) \quad \forall 0 \leq s \leq 1
$$

and its functional derivative

$$
-V \frac{\delta \mathscr{Q}[w]}{\delta w(\mathbf{r})}=\int_{0}^{1} \mathrm{~d} s q(\mathbf{r}, s) q(\mathbf{r}, 1-s) .
$$

For both chain models, the spatial dependency of the density is discretized using a one-dimensional grid with a typical resolution, $\Delta x=R_{\mathrm{e}} / 128$, for the bead-spring model and $\Delta x=R_{\mathrm{e}} / 128$ or $R_{\mathrm{e}} / 1024$ for the Gaussian-thread model.

\section{Acknowledgements}

Financial support by the DFG within the priority programme SPP 1369 "polymer-solid contacts: interfaces and interphases" (Mu1674/9) is gratefully acknowledged and we have benefited from computing time at the HLRN Hannover/Berlin and the Jülich Supercomputer Center.

\section{References}

1 A. Silberberg, J. Colloid Interface Sci., 1982, 90, 86.

2 D. T. Wu, G. H. Fredrickson, J. P. Carton, A. Adjdari and L. Leibler, J. Polym. Sci., Part B: Polym. Phys., 1995, 33, 2373.

3 A. N. Semenov, J. Phys. II, 1996, 6, 1759.

4 I. M. Lifshitz, A. Y. Grosberg and A. R. Khokhlov, Rev. Mod. Phys., 1978, 50, 683.

5 E. Helfand, J. Chem. Phys., 1975, 62, 999.

6 D. C. Morse and G. H. Fredrickson, Phys. Rev. Lett., 1994, 73, 3235.

7 F. Schmid and M. Müller, Macromolecules, 1995, 28, 8639.

8 M. W. Matsen, J. Chem. Phys., 1996, 104, 7758.

9 K. C. Daoulas, D. N. Theodorou, V. A. Harmandaris, N. C. Karayiannis and V. G. Mavrantzas, Macromolecules, 2005, 38, 7134.

10 E. Helfand and Y. Tagami, J. Polym. Sci., Part B: Polym. Lett., 1971, 9, 741.

11 J. M. H. M. Scheutjens and G. J. Fleer, J. Phys. Chem., 1979, 83, 1619.

12 J. M. H. M. Scheutjens and G. J. Fleer, J. Phys. Chem., 1980, 84, 178.

13 K. R. Shull, Macromolecules, 1992, 25, 2122.
14 J. Vavasour and M. Witmore, Macromolecules, 1993, 26, 7070.

15 M. Müller and F. Schmid, Adv. Polym. Sci., 2005, 185, 1.

16 M. W. Matsen, Soft Matter, ed. G. Gompper and M. Schick, 2006, vol. 1, p. 87.

17 A. N. Semenov, J. Bonet-Avalos, A. Johner and J. F. Joanny, Macromolecules, 1996, 29, 2179.

18 M. Müller, J. Chem. Phys., 2002, 116, 9930.

19 A. N. Semenov and A. Johner, Eur. Phys. J. E, 2003, 12, 469.

20 A. Cavallo, M. Müller, J. P. Wittmer, A. Johner and K. Binder, J. Phys.: Condens. Matter, 2005, 17, S1697.

21 M. Müller, K. Binder and L. Schäfer, Macromolecules, 2000, 33, 4568.

22 J. P. Wittmer, H. Meyer, J. Baschnagel, A. Johner, S. Obukhov, L. Mattioni, M. Müller and A. N. Semenov, Phys. Rev. Lett., 2004, 93, 147801.

23 J. Baschnagel, K. Binder, P. Doruker, A. A. Gusev, O. Hahn, K. Kremer, W. L. Mattice, F. Müller-Plathe, M. Murat and W. Paul, et al., Adv. Polym. Sci., 2000, 152, 41.

24 K. C. Daoulas and M. Müller, J. Chem. Phys., 2006, 125, 184904.

25 F. A. Detcheverry, H. M. Kang, K. C. Daoulas, M. Müller, P. F. Nealey and J. J. de Pablo, Macromolecules, 2008, 41, 4989.

26 F. A. Detcheverry, D. Q. Pike, P. F. Nealey, M. Müller and J. J. de Pablo, Phys. Rev. Lett., 2009, 102, 197801.

27 P. G. Bolhuis, A. A. Louis, J. P. Hansen and E. J. Meijer, J. Chem. Phys., 2001, 114, 4296.

28 A. Ramirez-Hernandez, F. A. Detcheverry and J. J. de Pablo, J. Chem. Phys., 2010, 133, 64905.

29 M. W. Matsen, Phys. Rev. Lett., 1995, 74, 4225.

30 K. M. Hong and J. Noolandi, Macromolecules, 1981, 14, 727.

31 M. W. Matsen and M. Schick, Phys. Rev. Lett., 1994, 72, 2660.

32 A. Ben-Shaul and I. Szleifer, J. Chem. Phys., 1985, 83, 3597.

33 I. Szleifer and M. A. Carignano, Adv. Chem. Phys., 1996, 94, 165.

34 M. Müller and A. Werner, J. Chem. Phys., 1997, 107, 10764.

35 M. Müller and M. Schick, Phys. Rev. E: Stat. Phys., Plasmas, Fluids, Relat. Interdiscip. Top., 1998, 57, 6973.

36 M. Müller and K. Binder, Macromolecules, 1998, 31, 8323.

37 K. Rasmussen and G. Kalosakas, J. Polym. Sci., Part B: Polym. Phys., 2002, 40, 1777.

38 F. Müller-Plathe, ChemPhysChem, 2002, 3, 754.

39 J.-P. Hansen and I. R. McDonald, Theory of Simple Liquids, Academic Press, London, 1986.

40 M. Müller and G. D. Smith, J. Polym. Sci., Part B: Polym. Phys., 2005, 43, 934.

41 K. C. Daoulas, M. Müller, J. J. de Pablo, P. F. Nealey and G. D. Smith, Soft Matter, 2006, 2, 573.

42 D. Q. Pike, F. A. Detcheverry, M. Müller and J. J. de Pablo, J. Chem. Phys., 2009, 131, 084903.

43 A. Yethiraj and C. E. Woodward, J. Chem. Phys., 1995, 102, 5499.

44 A. Yethiraj, J. Chem. Phys., 1998, 109, 3269.

45 A. Yethiraj, Adv. Chem. Phys., 2002, 121, 89.

46 Y. X. Yu and J. Z. Wu, J. Chem. Phys., 2002, 117, 2368.

47 M. Müller, L. G. MacDowell and A. Yethiraj, J. Chem. Phys., 2003, 118, 2929.

48 M. Müller and L. G. MacDowell, J. Phys.: Condens. Matter, 2003, 15, R609.

49 Y. X. Yu, J. Z. Wu, Y. X. Xin and G. H. Gao, J. Chem. Phys., 2004, 121, 1535.

50 J. Z. Wu, AIChE J., 2006, 52, 1169.

51 J. Z. Wu and Z. D. Li, Annu. Rev. Phys. Chem., 2007, $\mathbf{5 8}, 85$.

52 H. Kang, F. A. Detcheverry, A. N. Mangham, M. P. Stoykovich, K. C. Daoulas, R. J. Hamers, M. Müller, J. J. de Pablo and P. F. Nealey, Phys. Rev. Lett., 2008, 100, 148303.

53 M. Müller, L. G. MacDowell, P. Müller-Buschbaum, O. Wunnike and M. Stamm, J. Chem. Phys., 2001, 115, 9960.

54 The grand potential takes the simple form

$$
\frac{G}{k_{\mathrm{B}} T}=-\frac{\rho_{\text {bulk }}}{N} \int \mathrm{d} \mathbf{r} \pi(\phi(\mathbf{r}))
$$

with

$$
\pi(\phi)=\phi-f(\phi)+\phi \frac{\mathrm{d} f}{\mathrm{~d} \phi}=\phi+\frac{\kappa N}{2}\left(\phi^{2}-1\right) .
$$


Since $G_{\text {bulk }}=-p V$ in the homogeneous bulk, with $p$ being the pressure, the bulk equation of state is given by $p(\phi)=$ $k_{\mathrm{B}} T \rho_{\text {bulk }} \pi(\phi) / N$ and the mean-field approximation of the interface tension, $\gamma$, can be calculated as the spatial integral of the deviation of the pressure from its bulk value

$$
\frac{\gamma R_{\mathrm{e}}^{2}}{k_{\mathrm{B}} T \sqrt{\bar{N}}}=\int_{0}^{D / 2} \frac{\mathrm{d} x}{R_{\mathrm{e}}}\left[\pi\left(\phi_{\text {bulk }}\right)-\pi(\phi(x))\right] .
$$

55 The occurrence of cluster crystals at high densities can be avoided by a judicious choice of $w, c f$. C. N. Likos, B. M. Mladek, D. Gottwald and G. Kahl, J. Chem. Phys., 2007, 126, 224502.

56 Due to the small discretization, $N=32$, the data for the beadspring model rather correspond to an extended "end-fraction" of a molecule, which exhibitsa less pronounced spatial variation of the relative excess.

57 In order to perform calculations in the canonical ensemble we have augmented the self-consistent set of equations, (12) and 13), by a single equation, $\zeta \mathscr{Q}=1$, and adjusted both the field $w(x)$ and the excess activity, $\zeta$. Since the normalized density at the center of the film is larger than unity, $\zeta>1$ in the canonical calculations. For the Gaussian-thread model we obtain $\zeta=8.095$ for film thickness $D=4 R_{\mathrm{e}}$. Altering the bonded interactions in the vicinity of the polymer-solid contact, we obtain $\zeta=6.856$ for the bead-spring model. Using an external segment-solid interaction, we can interpret the plateau value $c=\kappa N\left(\phi_{\text {Gauss,can }}-1\right)-W$ at the center of the film as a shift of the chemical potential and obtain $\zeta=\exp (c)=8.093$. Thus the external segment-solid interaction also improves the thermodynamic predictions (e.g., polymer adsorption/partitioning into confined environments) of the coarse-grained model.

58 This Dirichlet boundary condition will be appropriate if the spatial discretization is sufficient to resolve the spatial variation of $w(x)$. This condition is fulfilled in our calculations. For the case that the self-consistent field calculations of Gaussian threads cannot resolve the spatial dependence of the interactions, effective boundary conditions for eqn (37) have been devised, $c f$. G. H. Fredrickson, The Equilibrium Theory of Inhomogeneous Polymers, Clarendon Press, Oxford, 2006. 